\title{
A framework for generalized conjugate gradient methods-with special emphasis on contributions by Rüdiger Weiss
}

\author{
Martin H. Gutknecht ${ }^{\mathrm{a}, *}$, Miroslav Rozložník ${ }^{\mathrm{b}, 1}$ \\ ${ }^{a}$ Seminar for Applied Mathematics, ETH-Zentrum HG, CH-8092 Zürich, Switzerland \\ ${ }^{\mathrm{b}}$ Institute of Computer Science, Academy of Sciences of the Czech Republic, Pod vodárenskou věží 2, \\ CZ-18207 Prague 8, Czech Republic
}

Dedicated to the memory of Rüdiger Weiss

\begin{abstract}
We discuss a general framework for generalized conjugate gradient methods, that is, iterative methods (for solving linear systems) that are based on generating residuals that are formally orthogonal to each other with respect to some true or formal inner product. This includes methods that generate residuals that are minimal with respect to some norm based on an inner product. A similar, even more general framework was introduced and extensively discussed by Weiss in his work.

Weiss also emphasized in his work the relationship between certain pairs of orthogonal and minimal residual methods, where the results of the second method can be generated from those of the first method by applying the minimal residual smoothing process. Examples of such pairs are the conjugate gradient (CG) and the conjugate residual (CR) methods, the full orthogonalization method (FOM) and the generalized minimal residual (GMRES) method, as well as the CGNE and CGNR versions of applying CG to the normal equations. () 2001 IMACS. Published by Elsevier Science B.V. All rights reserved.
\end{abstract}

Keywords: Sparse linear systems; Krylov space method; Orthogonal residual method; Minimal residual method; Conjugate gradient method; Residual smoothing; CG; CGNE; CGNR; CR; FOM; GMRES; PRES

\section{Introduction}

We consider the iterative solution of nonsingular real linear $N \times N$ systems $\boldsymbol{A} \boldsymbol{x}=\boldsymbol{b}$ by certain Krylov space methods, which can be defined by an orthogonality condition for the residuals. Such methods

\footnotetext{
* Corresponding author.

E-mail addresses: mhg@ sam.math.ethz.ch (M.H. Gutknecht), miro@ cs.cas.cz (M. Rozložník).

URL address: http://www.sam.math.ethz.ch/ mhg.

${ }^{1}$ Part of this author's work was supported by the Grant Agency of the Czech Republic under grant No. 101/00/1035 and by the Grant Agency of the Academy of Sciences of the Czech Republic grant No. A1030103.
} 
are sometimes vaguely referred to as generalized conjugate gradient methods or conjugate-gradient-type methods. More specific are the notions of orthogonal residual methods and minimal residual methods (or, minimum residual methods). We discuss a framework for such methods that was introduced by Rüdiger Weiss in his Ph.D. thesis [37] and was further developed in some of his papers, most notably in [38], and in his book [41]. We partly follow and explain his argumentation, but we consider a somewhat less general, but more specific framework and look at it also from a different perspective.

We let $\boldsymbol{x}_{0}$ and $\boldsymbol{r}_{0}: \equiv \boldsymbol{b}-\boldsymbol{A} \boldsymbol{x}_{0}$ be an initial guess of the solution and the corresponding residual, respectively (often $\boldsymbol{x}_{0}=\boldsymbol{o}$ and $\boldsymbol{r}_{0}=\boldsymbol{b}$ ). Most Krylov space methods generate a sequence of approximations or iterates $\boldsymbol{x}_{n}$ with the property that

$$
\boldsymbol{x}_{n}-\boldsymbol{x}_{0} \in \mathcal{K}_{n}\left(\boldsymbol{A}, \boldsymbol{r}_{0}\right): \equiv \operatorname{span}\left\{\boldsymbol{r}_{0}, \boldsymbol{A} \boldsymbol{r}_{0}, \ldots, \boldsymbol{A}^{n-1} \boldsymbol{r}_{0}\right\} .
$$

Here, $\mathcal{K}_{n}\left(\boldsymbol{A}, \boldsymbol{r}_{0}\right)$ is the Krylov space generated by $\boldsymbol{A}$ from $\boldsymbol{r}_{0}$. There are also Krylov space methods using different spaces, like $\mathcal{K}_{n}\left(\boldsymbol{A}^{\top}, \boldsymbol{r}_{0}\right)$ or $\mathcal{K}_{n}\left(\boldsymbol{A} \boldsymbol{A}^{\top}, \boldsymbol{r}_{0}\right)$, as we will see.

We start here from a more general situation and let $\left\{\mathcal{L}_{n}\right\}_{n=1}^{m}$ be a finite nested sequence of subspaces, so that $\operatorname{dim} \mathcal{L}_{n}=n$ for $n \leqslant m$ and $\operatorname{dim} \mathcal{L}_{m+1}=m$. In other words, for $n \leqslant m$

$$
\mathcal{L}_{n}: \equiv \operatorname{span}\left\{\boldsymbol{y}_{0}, \boldsymbol{y}_{1}, \ldots, \boldsymbol{y}_{n-1}\right\}
$$

with linearly independent (basis) vectors $\boldsymbol{y}_{0}, \boldsymbol{y}_{1}, \ldots, \boldsymbol{y}_{m-1}$ and a linearly dependent $\boldsymbol{y}_{m}$. We further assume that $\boldsymbol{x}_{n}-\boldsymbol{x}_{0} \in \mathcal{L}_{n}$, that is, in our notation,

$$
\boldsymbol{x}_{n} \in \boldsymbol{x}_{0}+\mathcal{L}_{n} .
$$

The residuals and errors associated with $\boldsymbol{x}_{n}$ are, respectively,

$$
\boldsymbol{r}_{n}: \equiv \boldsymbol{b}-\boldsymbol{A} \boldsymbol{x}_{n}, \quad \boldsymbol{e}_{n}: \equiv \boldsymbol{x}_{n}-\boldsymbol{A}^{-1} \boldsymbol{b} .
$$

Clearly, (3) implies that

$$
\boldsymbol{r}_{n} \in \boldsymbol{r}_{0}+\boldsymbol{A} \mathcal{L}_{n}, \quad \boldsymbol{e}_{n} \in \boldsymbol{e}_{0}+\mathcal{L}_{n} .
$$

The methods to be discussed here are based on an orthogonality condition for the residuals,

$$
\left\langle\boldsymbol{r}_{n}, \boldsymbol{Z} \boldsymbol{y}\right\rangle=0\left(\forall \boldsymbol{y} \in \mathcal{L}_{n}\right) \quad \text { or, briefly, } \quad \boldsymbol{r}_{n} \perp_{\boldsymbol{Z}} \mathcal{L}_{n},
$$

where $\boldsymbol{Z}$ is a formal inner product matrix, which may be or may not be symmetric positive definite (spd). If it is, we have a true inner product. The orthogonality condition (6) has a simple consequence, which plays an important role in Weiss' work:

Lemma 1. For any iterative method satisfying (2)-(4) and (6) holds

$$
\boldsymbol{r}_{n} \perp_{\boldsymbol{Z}} \boldsymbol{A}^{-1}\left(\boldsymbol{r}_{n}-\boldsymbol{r}_{0}\right)=\boldsymbol{e}_{0}-\boldsymbol{e}_{n} .
$$

Proof. The condition (3) implies that $\boldsymbol{e}_{n} \in \boldsymbol{e}_{0}+\mathcal{L}_{n}$. Moreover, by (4), $\boldsymbol{r}_{n}=-\boldsymbol{A} \boldsymbol{e}_{n}(\forall n)$. Thus $\boldsymbol{A}^{-1}\left(\boldsymbol{r}_{n}-\right.$ $\left.\boldsymbol{r}_{0}\right)=\boldsymbol{e}_{0}-\boldsymbol{e}_{n} \in \mathcal{L}_{n}$, and the claim follows from (6).

Clearly, the standard Krylov space solvers satisfying (1) are covered by setting

$$
\mathcal{L}_{n}:=\mathcal{K}_{n}\left(\boldsymbol{A}, \boldsymbol{r}_{0}\right) \text {. }
$$

If $\boldsymbol{Z}$ is a (finite) spd matrix, or if $\boldsymbol{A}$ is a (finite) nonsingular matrix and $\boldsymbol{Z}=\boldsymbol{A}$, any solver satisfying (3)-(4) and (6)-(7) will converge (in exact arithmetic) in a finite number of steps unless it breaks down 
before, that is, it has the finite termination property. In fact, due to the Caley-Hamilton theorem, there is some finite $m$ such that $\mathcal{K}_{m+1}\left(\boldsymbol{A}, \boldsymbol{r}_{0}\right)=\mathcal{K}_{m}\left(\boldsymbol{A}, \boldsymbol{r}_{0}\right)$, and we can conclude using (6) that

$$
\mathcal{K}_{m}\left(\boldsymbol{A}, \boldsymbol{r}_{0}\right) \perp_{\boldsymbol{Z}} \boldsymbol{r}_{m} \in \mathcal{K}_{m+1}\left(\boldsymbol{A}, \boldsymbol{r}_{0}\right)=\mathcal{K}_{m}\left(\boldsymbol{A}, \boldsymbol{r}_{0}\right) \Longrightarrow \boldsymbol{r}_{m}=\boldsymbol{o} .
$$

(Under the second assumption mentioned $\mathcal{K}_{m}\left(\boldsymbol{A}, \boldsymbol{r}_{0}\right) \perp \boldsymbol{A} \boldsymbol{r}_{m} \in \mathcal{K}_{m+1}\left(\boldsymbol{A}, \boldsymbol{r}_{0}\right)=\mathcal{K}_{m}\left(\boldsymbol{A}, \boldsymbol{r}_{0}\right)$, and we can conclude that $\boldsymbol{A} \boldsymbol{r}_{m}=\boldsymbol{o}$, so $\boldsymbol{r}_{m}=\boldsymbol{o}$ due to the nonsingularity of $\boldsymbol{A}$.) There are yet other cases where the finite termination property holds.

For finding iterates and residuals satisfying (2)-(6), we could apply the Gram-Schmidt process as follows. A proof is easily found by adapting the one of the similar result in Section 4.3 of [18].

Theorem 2. If defined for all $n$ up to $m$, the iterates $\boldsymbol{x}_{n}$ and the residuals $\boldsymbol{r}_{n}$ of a Krylov space solver characterized by (1) satisfy, for $n=0,1, \ldots, m-1$,

$$
\begin{aligned}
\boldsymbol{x}_{n+1} & :=-\left(\boldsymbol{y}_{n}+\boldsymbol{x}_{n} \eta_{n, n}^{\circ}+\boldsymbol{x}_{n-1} \eta_{n-1, n}^{\circ}+\cdots+\boldsymbol{x}_{0} \eta_{0, n}^{\circ}\right) / \eta_{n+1, n}^{\circ}, \\
\boldsymbol{r}_{n+1} & :=\left(\boldsymbol{A} \boldsymbol{y}_{n}-\boldsymbol{r}_{n} \eta_{n, n}^{\circ}-\boldsymbol{r}_{n-1} \eta_{n-1, n}^{\circ}-\cdots-\boldsymbol{r}_{0} \eta_{0, n}^{\circ}\right) / \eta_{n+1, n}^{\circ},
\end{aligned}
$$

where $\eta_{0, n}^{\circ}, \ldots, \eta_{n, n}^{\circ}$ are chosen such that $\boldsymbol{r}_{n+1} \perp_{Z} \mathcal{L}_{n+1}$ and where

$$
\eta_{n+1, n}^{\circ}: \equiv-\eta_{n, n}^{\circ}-\eta_{n-1, n}^{\circ}-\cdots-\eta_{0, n}^{\circ} .
$$

In terms of the matrices

$$
\boldsymbol{Y}_{m}: \equiv\left[\boldsymbol{y}_{0} \cdots \boldsymbol{y}_{m-1}\right], \quad \boldsymbol{X}_{m}: \equiv\left[\boldsymbol{x}_{0} \cdots \boldsymbol{x}_{m-1}\right], \quad \boldsymbol{R}_{m}: \equiv\left[\boldsymbol{r}_{0} \cdots \boldsymbol{r}_{m-1}\right],
$$

and $\underline{\boldsymbol{H}}_{m}^{\circ}: \equiv\left(\eta_{k, l}^{\circ}\right)$, the recursions (9)-(10) can be expressed as

$$
\boldsymbol{Y}_{m}=-\boldsymbol{X}_{m+1} \underline{\boldsymbol{H}}_{m}^{\circ}, \quad \boldsymbol{A} \boldsymbol{Y}_{m}=\boldsymbol{R}_{m+1} \underline{\boldsymbol{H}}_{m}^{\circ},
$$

where $\underline{\boldsymbol{H}}_{m}^{\circ}$ is an $(m+1) \times m$ Hessenberg matrix with column sums 0 .

In the case of a Krylov space solver characterized by (1), where $\mathcal{L}_{n}:=\mathcal{K}_{n}\left(\boldsymbol{A}, \boldsymbol{r}_{0}\right)$, we could let $\boldsymbol{y}_{n}:=\boldsymbol{A}^{n-1} \boldsymbol{r}_{0}$ in (2) and enforce (6) by $\boldsymbol{Z}$-orthogonalization of this basis. However, it is well known that the basis $\left\{\boldsymbol{A}^{k} \boldsymbol{r}_{0}\right\}_{k=0}^{m-1}$ is so badly conditioned that this does not work in floating point arithmetic. Instead one applies recursive procedures to build up the space, by choosing $\boldsymbol{y}_{n}:=\boldsymbol{r}_{n} v_{n}$ for some scalars $v_{n}$. If $\boldsymbol{A}$ is symmetric, this can be a variation of the symmetric Lanczos process, otherwise one of the Arnoldi process. Such a process may at the same time directly generate the residuals $\boldsymbol{r}_{n}$ and, with little extra work also the iterates $\boldsymbol{x}_{n}$.

More generally, we could aim at a Krylov space generated by some nonsingular matrix $\boldsymbol{B}$ different from $\boldsymbol{A}$ and let

$$
\mathcal{L}_{n}:=\boldsymbol{A}^{-1} \boldsymbol{B} \mathcal{K}_{n}\left(\boldsymbol{B}, \boldsymbol{r}_{0}\right)=\operatorname{span}\left\{\boldsymbol{A}^{-1} \boldsymbol{B} \boldsymbol{r}_{0}, \boldsymbol{A}^{-1} \boldsymbol{B}^{2} \boldsymbol{r}_{0}, \ldots, \boldsymbol{A}^{-1} \boldsymbol{B}^{n} \boldsymbol{r}_{0}\right\}
$$

Then (3) still implies (5), and in the recursions (9)-(10) we can choose

$$
\boldsymbol{y}_{n}: \equiv \boldsymbol{A}^{-1} \boldsymbol{B} \boldsymbol{r}_{n},
$$

so that they become 


$$
\begin{aligned}
\boldsymbol{x}_{n+1} & :=-\left(\boldsymbol{A}^{-1} \boldsymbol{B} \boldsymbol{r}_{n}+\boldsymbol{x}_{n} \eta_{n, n}^{\circ}+\boldsymbol{x}_{n-1} \eta_{n-1, n}^{\circ}+\cdots+\boldsymbol{x}_{0} \eta_{0, n}^{\circ}\right) / \eta_{n+1, n}^{\circ}, \\
\boldsymbol{r}_{n+1} & :=\left(\boldsymbol{B} \boldsymbol{r}_{n}-\boldsymbol{r}_{n} \eta_{n, n}^{\circ}-\boldsymbol{r}_{n-1} \eta_{n-1, n}^{\circ}-\cdots-\boldsymbol{r}_{0} \eta_{0, n}^{\circ}\right) / \eta_{n+1, n}^{\circ} .
\end{aligned}
$$

In the standard case of $\boldsymbol{B}=\boldsymbol{A}$ they reduce further to

$$
\begin{aligned}
\boldsymbol{x}_{n+1} & :=-\left(\boldsymbol{r}_{n}+\boldsymbol{x}_{n} \eta_{n, n}^{\circ}+\boldsymbol{x}_{n-1} \eta_{n-1, n}^{\circ}+\cdots+\boldsymbol{x}_{0} \eta_{0, n}^{\circ}\right) / \eta_{n+1, n}^{\circ}, \\
\boldsymbol{r}_{n+1} & :=\left(\boldsymbol{A} \boldsymbol{r}_{n}-\boldsymbol{r}_{n} \eta_{n, n}^{\circ}-\boldsymbol{r}_{n-1} \eta_{n-1, n}^{\circ}-\cdots-\boldsymbol{r}_{0} \eta_{0, n}^{\circ}\right) / \eta_{n+1, n}^{\circ} .
\end{aligned}
$$

Clearly, we have to assume that there is an easy way to compute $\boldsymbol{A}^{-1} \boldsymbol{B} \boldsymbol{r}_{n}$. This is certainly the case when $\boldsymbol{B}=\boldsymbol{A}$ or $\boldsymbol{B}=\boldsymbol{A} \boldsymbol{A}^{\top}$. The formulas (18)-(19) represent the ORTHORES form of a Krylov space solver.

If $\boldsymbol{B}=\boldsymbol{A}$ and if the residuals are orthogonal (with respect to an spd matrix $\boldsymbol{Z}$ ), as implied by (6), such a method is often called an orthogonal residual method, sometimes also a Galerkin method or an orthogonal projection method.

Schönauer and Weiss call this a pseudo-residual method or a PRES method, because the numerator in (19), the "pseudo-residual"

$$
\overline{\boldsymbol{r}}_{n+1}: \equiv \boldsymbol{A} \boldsymbol{r}_{n}-\boldsymbol{r}_{n} \eta_{n, n}^{\circ}-\boldsymbol{r}_{n-1} \eta_{n-1, n}^{\circ}-\cdots-\boldsymbol{r}_{0} \eta_{0, n}^{\circ}=\boldsymbol{r}_{n+1} \eta_{n+1, n}^{\circ}
$$

is minimized in the $\boldsymbol{Z}$-norm:

$$
\left\|\overline{\boldsymbol{r}}_{n+1}\right\|_{\boldsymbol{Z}}=\min ! \quad \text { subject to } \boldsymbol{x}_{n+1} \in \boldsymbol{x}_{0}+\mathcal{L}_{n+1} \text {. }
$$

In fact, this minimization clearly requires that $\overline{\boldsymbol{r}}_{n+1}$ be $\boldsymbol{Z}$-orthogonal to $\boldsymbol{r}_{0}, \boldsymbol{r}_{1}, \ldots, \boldsymbol{r}_{n}$, so that (6) holds. The orthogonality condition then determines the values of the recurrence coefficients $\eta_{0, n}^{\circ}, \ldots, \eta_{n, n}^{\circ}$, but it does not mean that the residual itself is minimal in some sense.

In contrast, a minimal residual method or conjugate residual method is one where the residual is minimized (in the $\boldsymbol{Z}$-norm);

$$
\left\|\boldsymbol{r}_{n+1}\right\|_{\boldsymbol{Z}}=\min ! \quad \text { subject to } \boldsymbol{x}_{n+1} \in \boldsymbol{x}_{0}+\mathcal{L}_{n+1} \text {. }
$$

There is also an orthogonality condition (Galerkin condition) associated with this minimization problem. It will be discussed below. We note that both (20) and (21) make sense in the more general setting of (2).

In practice, Krylov space solvers are only useful if they involve only a limited number of previous iterates and residuals. Preferable are those where this number is small—one or two- that is, solvers with short recurrences. For some methods, like CG, CR, BICG, this holds due to their particular mathematical properties. For other methods, it is enforced by truncation or restarting, but then we may need to include on the order of 10 or even many more previous residuals. Weiss combined all these cases in one set of formulas replacing (18)-(19) (we leave out the preconditioning he included, and adapt to our notation in (18)-(19)):

$$
\begin{aligned}
\boldsymbol{x}_{n+1} & :=-\left(\boldsymbol{r}_{n}+\sum_{i=1}^{\iota_{n}} \boldsymbol{x}_{n+1-i} \eta_{n+1-i, n}^{\circ}\right) / \eta_{n+1, n}^{\circ}, \\
\overline{\boldsymbol{r}}_{n+1} & :=\boldsymbol{A} \boldsymbol{r}_{n}-\sum_{i=1}^{\iota_{n}} \boldsymbol{r}_{n+1-i} \eta_{n+1-i, n}^{\circ}, \\
\boldsymbol{r}_{n+1} & :=\overline{\boldsymbol{r}}_{n+1} / \eta_{n+1, n}^{\circ} .
\end{aligned}
$$

He then classified methods according to the following list in Table 1. 
Table 1

\begin{tabular}{ll}
\hline Type of method & Condition for $\iota_{n}$ \\
\hline exact & $\iota_{n}=n+1(\forall n)$ \\
restarted & $\iota_{n}=n \bmod \iota_{\text {restart }}+1$ \\
truncated & $\iota_{n}=\min \left\{n+1, \iota_{\max }\right\}$ \\
combined & truncated method is restarted \\
\hline
\end{tabular}

Among the other ways to implement these methods is, in particular, the ORTHOMIN form, which constructs additionally direction vectors $\boldsymbol{v}_{n}$ so that for some scalar $\omega_{n}$

$$
\boldsymbol{x}_{n+1}:=\boldsymbol{x}_{n}+\boldsymbol{v}_{n} \omega_{n} .
$$

The ORTHOMIN recurrence coefficients are related to those of ORTHORES by an LU decomposition of the matrix $\underline{\boldsymbol{H}}_{m}^{\circ}$, see [1,17]. There is also a general ORTHODIR recurrence for the same methods; see $[42,1]$.

\section{Geometric properties of generalized conjugate gradient methods}

In this section we concentrate on some geometric properties of generalized conjugate gradient methods discussed by Weiss in $[37,38,41]$. We consider first our general setting (3) with (2), but then we concentrate on (1), that is, (3) with (7). We examine the two main classes of generalized conjugate gradient methods characterized by (20) and (21), respectively: orthogonal residual methods and minimal residual methods. We give examples of such methods and discuss their theoretical properties in a similar, but slightly different fashion as in $[38,41]$.

We then also show that the CGNR and CGNE versions of applying the CG algorithm to normal equations fit into our general framework for Krylov space solvers allowing for $\boldsymbol{B} \neq \boldsymbol{A}$. We capitalize upon these results and show how this analysis leads to the notion of minimal error methods. These will be examined further in the next section.

It is easy to verify by computing the Fréchet-derivative of $\left\|\boldsymbol{r}_{n+1}\right\|_{Z}$ that in the general framework based on (2)-(3) minimal residual methods characterized by (21) satisfy the orthogonality condition (6) with $\boldsymbol{Z}=\boldsymbol{A}$, so that the statement of Lemma 1 can be rewritten as follows.

Corollary 3. For any minimal residual methods characterized by (21) with (2) or, equivalently, by

$$
\boldsymbol{r}_{n} \perp_{\boldsymbol{A}} \mathcal{L}_{n} \quad \text { subject to } \boldsymbol{x}_{n} \in \boldsymbol{x}_{0}+\mathcal{L}_{n},
$$

the residual vector $\boldsymbol{r}_{n}$ and the error vector $\boldsymbol{e}_{n}$ satisfy the following relations:

$$
\begin{aligned}
& \left\langle\boldsymbol{r}_{n}, \boldsymbol{r}_{n}-\boldsymbol{r}_{0}\right\rangle=0, \\
& \left\|\boldsymbol{r}_{n}\right\|^{2}+\left\|\boldsymbol{r}_{n}-\boldsymbol{r}_{0}\right\|^{2}=\left\|\boldsymbol{r}_{0}\right\|^{2}, \\
& \left\|\boldsymbol{e}_{n}\right\|_{\boldsymbol{A}^{\top} \boldsymbol{A}}^{2}+\left\|\boldsymbol{e}_{n}-\boldsymbol{e}_{0}\right\|_{\boldsymbol{A}^{\top} \boldsymbol{A}}^{2}=\left\|\boldsymbol{e}_{0}\right\|_{\boldsymbol{A}^{\top} \boldsymbol{A}}^{2} .
\end{aligned}
$$

The relation (23) has an interpretation in terms of the Thales theorem: the residual vector $\boldsymbol{r}_{n}$ lies on an $n$-dimensional sphere whose diameter is equal to the norm of the initial residual $\boldsymbol{r}_{0}$; see Fig. 1 . As a 

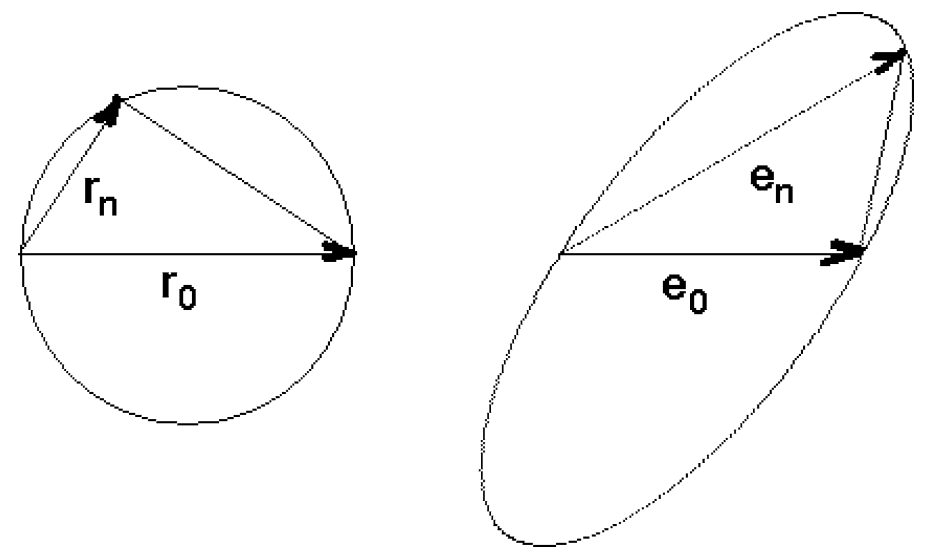

Fig. 1. Geometric properties of minimal residual methods.

consequence, (24) is just the statement of Pythagoras' theorem, and (25) is its reformulation for the error $\boldsymbol{e}_{n}$ in terms of its $\boldsymbol{A}^{\top} \boldsymbol{A}$-norm.

Several methods that fit into our general class of minimal residual methods have been proposed and used. The most important one minimizes the 2-norm of the residual with respect to the Krylov space (7) generated by $\boldsymbol{A}$ from $\boldsymbol{r}_{0}$; in this case

$$
\boldsymbol{r}_{n} \perp_{\boldsymbol{A}} \mathcal{K}_{n}\left(\boldsymbol{A}, \boldsymbol{r}_{0}\right) \quad \text { subject to } \boldsymbol{x}_{n} \in \boldsymbol{x}_{0}+\mathcal{K}_{n}\left(\boldsymbol{A}, \boldsymbol{r}_{0}\right) \text {. }
$$

By definition it satisfies

$$
\begin{aligned}
& \left\|\boldsymbol{b}-\boldsymbol{A} \boldsymbol{x}_{n}\right\|=\min _{\boldsymbol{x} \in \boldsymbol{x}_{0}+\mathcal{K}_{n}\left(\boldsymbol{A}, \boldsymbol{r}_{0}\right)}\|\boldsymbol{b}-\boldsymbol{A} \boldsymbol{x}\|, \\
& \left\|\boldsymbol{x}_{n}-\boldsymbol{A}^{-1} \boldsymbol{b}\right\|_{\boldsymbol{A}^{\top} \boldsymbol{A}}=\min _{\boldsymbol{x} \in \boldsymbol{x}_{0}+\mathcal{K}_{n}\left(\boldsymbol{A}, \boldsymbol{r}_{0}\right)}\left\|\boldsymbol{x}-\boldsymbol{A}^{-1} \boldsymbol{b}\right\|_{\boldsymbol{A}^{\top} \boldsymbol{A}} .
\end{aligned}
$$

The approximate solution $\boldsymbol{x}_{n}$ satisfying these conditions always exists, and it is uniquely determined. In fact, it is easy to see that for $n<m,(27)$ is a full-rank least squares problem, while for $n \geqslant m$ (when the Krylov space is exhausted) the solution is $\boldsymbol{x}_{n}=\boldsymbol{A}^{-1} \boldsymbol{b}$, that is, the exact solution has been found, and the residual norm is zero. Note that $\boldsymbol{A}$-orthogonal residuals are, of course, linearly independent. Thus, it is also clear from (27) and (28) that the 2-norm of the residual and the $\boldsymbol{A}^{\top} \boldsymbol{A}$-norm of the error converge monotonously.

Numerous variants and schemes with many different names have been proposed for this method. Although, in exact arithmetic, they all generate the same approximate solutions $\boldsymbol{x}_{n}$, they differ in various algorithmic aspects, and hence their behavior in floating point arithmetic can differ substantially.

In the case of a symmetric positive definite matrix $\boldsymbol{A}$ the first residual norm minimizing method was the conjugate residual (CR) method of Stiefel [31], which is just the conjugate gradient method with the energy inner product. Both the original coupled two-term (OMIN) implementation of CR and the three-term (ORES) implementation that fits into the pattern of the general recursions (18)-(19) can break down if $\boldsymbol{A}$ is not positive definite [2]. But the solution $\boldsymbol{x}_{n}$ of (27) is well-defined even in this case. In fact, the less well-known and either less stable or more expensive ODIR version of CR works for any symmetric matrix $\boldsymbol{A}$. Other variations of CR for indefinite symmetric systems have been proposed by Luenberger [24], Fletcher [13], and Chandra (MCR algorithm) [5]. A different approach took Paige 
and Saunders [25] with their MINRES algorithm, which applies the symmetric Lanczos process for constructing an orthonormal basis of (7) and then solves the minimal residual problem in the coordinate space with respect to this basis. Since the basis is orthonormal, the coordinate mapping is an isometry, that is, the 2-norm of the residual is equal to the 2-norm of its coordinates.

The first minimal residual method for nonsymmetric systems was derived by Khabaza [22]. Another approach, the generalized conjugate gradient (GCG) method, was proposed by Axelsson in [3]. Closely related to Axelsson's method are the ORTHOMIN [33] and the generalized conjugate residual (GCR) methods [11], which may not converge for systems which are not positive real, however. The more generally applicable ORTHODIR method was proposed by Young and Jea [42]. But the best-known and most frequently used algorithm is the generalized minimal residual (GMRES) method of Saad and Schultz [29], which, in full analogy to MINRES is based on the explicit computation of an orthogonal basis of (7) by the Arnoldi process and the solution of the minimum problem in the coordinate space. Several other implementations and variants of GMRES have been proposed including some with stable and efficient orthogonalization techniques; see [34,26,36].

The second large class of generalized conjugate gradient methods is in the framework (2)-(6) given by the choice $\boldsymbol{Z}=\boldsymbol{I}$, which describes the Galerkin condition

$$
\boldsymbol{r}_{n} \perp \mathcal{L}_{n} \quad \text { subject to } \boldsymbol{x}_{n} \in \boldsymbol{x}_{0}+\mathcal{L}_{n} .
$$

In the case of a classical Krylov space solver (1) this becomes

$$
\boldsymbol{r}_{n} \perp \mathcal{K}_{n}\left(\boldsymbol{A}, \boldsymbol{r}_{0}\right) \quad \text { subject to } \boldsymbol{x}_{n} \in \boldsymbol{x}_{0}+\mathcal{K}_{n}\left(\boldsymbol{A}, \boldsymbol{r}_{0}\right) .
$$

Since, in this situation, we have $\boldsymbol{r}_{k} \in \boldsymbol{r}_{0}+\boldsymbol{A} \mathcal{L}_{k} \subseteq \mathcal{L}_{n}$ when $k<n$, the methods characterized by (29) are called orthogonal residual methods. Unfortunately, for general nonsymmetric system and even for symmetric indefinite ones, the approximate solution satisfying (29) need not exist at all steps $n$. This potential hazard makes these methods less attractive to many users, although there is a simple way to get around this problem, namely by keeping track of the normalization of the residual polynomial separately; see, e.g., [18] for the corresponding adjustment of the biconjugate gradient method, which encounters the same problem.

There is a strong connection between orthogonal residual methods and minimal residual methods, which was discovered by Weiss in his thesis [37] and will be discussed later in this paper.

For symmetric positive definite $\boldsymbol{A}$ the orthogonal residual method has the important features that the approximate solution $\boldsymbol{x}_{n}$ exists at each iteration step and that the Galerkin condition (30) is equivalent to each of the following two minimization properties

$$
\begin{aligned}
& \left\|\boldsymbol{b}-\boldsymbol{A} \boldsymbol{x}_{n}\right\|_{\boldsymbol{A}^{-1}}=\min _{\boldsymbol{x} \in \boldsymbol{x}_{0}+\mathcal{K}_{n}\left(\boldsymbol{A}, \boldsymbol{r}_{0}\right)}\|\boldsymbol{b}-\boldsymbol{A} \boldsymbol{x}\|_{\boldsymbol{A}^{-1}}, \\
& \left\|\boldsymbol{x}_{n}-\boldsymbol{A}^{-1} \boldsymbol{b}\right\|_{\boldsymbol{A}}=\min _{\boldsymbol{x} \in \boldsymbol{x}_{0}+\mathcal{K}_{n}\left(\boldsymbol{A}, \boldsymbol{r}_{0}\right)}\left\|\boldsymbol{x}-\boldsymbol{A}^{-1} \boldsymbol{b}\right\|_{\boldsymbol{A}} .
\end{aligned}
$$

The equivalent conditions (31) and (32) are characteristic properties of the most popular iterative scheme, which has also initiated all the research on its many generalizations - the conjugate gradient (CG) method due to Hestenes and Stiefel [21]. Since the CG method is restricted to symmetric positive definite systems, several other orthogonal residual methods for solving symmetric indefinite or nonsymmetric systems have been proposed. Golub, Concus and Widlund [6] presented what is now called the GCW method for systems with shifted skew-symmetric matrices. Special forms of the GCG and the ORTHORES algorithms were studied by Hageman and Young [20] and by Elman [12]. The full orthogonalization 
method (FOM) [28], also called the Arnoldi method [4] since based on the Arnoldi orthogonalization process, is an orthogonal residual method for general nonsymmetric problems.

Another generalization of the CG method to general nonsymmetric systems that fits into the class of orthogonal residual methods is the CG method applied to one of the two positive definite systems of normal equations. The CG method applied to the normal equations $\boldsymbol{A}^{\top} \boldsymbol{A} \boldsymbol{x}=\boldsymbol{A}^{\top} \boldsymbol{b}$ is known as the CGNR method. It was already proposed by Hestenes and Stiefel [21]. In view of the properties of the CG method (now applied to the system of normal equations with the positive definite matrix $\boldsymbol{A}^{\top} \boldsymbol{A}$ ) the approximate solutions $\boldsymbol{x}_{n}$ of the CGNR method satisfy in the case of a real matrix the conditions

$$
\begin{aligned}
& \boldsymbol{x}_{n} \in \boldsymbol{x}_{0}+\mathcal{K}_{n}\left(\boldsymbol{A}^{\top} \boldsymbol{A}, \boldsymbol{A}^{\top} \boldsymbol{r}_{0}\right)=\boldsymbol{x}_{0}+\boldsymbol{A}^{\top} \mathcal{K}_{n}\left(\boldsymbol{A} \boldsymbol{A}^{\top}, \boldsymbol{r}_{0}\right), \\
& \boldsymbol{r}_{n} \in \boldsymbol{r}_{0}+\boldsymbol{A} \mathcal{K}_{n}\left(\boldsymbol{A}^{\top} \boldsymbol{A}, \boldsymbol{A}^{\top} \boldsymbol{r}_{0}\right)=\boldsymbol{r}_{0}+\boldsymbol{A} \boldsymbol{A}^{\top} \mathcal{K}_{n}\left(\boldsymbol{A} \boldsymbol{A}^{\top}, \boldsymbol{r}_{0}\right), \\
& \boldsymbol{r}_{n} \perp_{\boldsymbol{A}} \mathcal{K}_{n}\left(\boldsymbol{A}^{\top} \boldsymbol{A}, \boldsymbol{A}^{\top} \boldsymbol{r}_{0}\right)=\boldsymbol{A}^{\top} \mathcal{K}_{n}\left(\boldsymbol{A} \boldsymbol{A}^{\top}, \boldsymbol{r}_{0}\right),
\end{aligned}
$$

which, by letting $\boldsymbol{B}: \equiv \boldsymbol{A} \boldsymbol{A}^{\top}$, may also be written as

$$
\begin{aligned}
& \boldsymbol{x}_{n} \in \boldsymbol{x}_{0}+\boldsymbol{A}^{-1} \boldsymbol{B} \mathcal{K}_{n}\left(\boldsymbol{B}, \boldsymbol{r}_{0}\right), \\
& \boldsymbol{r}_{n} \in \boldsymbol{r}_{0}+\boldsymbol{B} \mathcal{K}_{n}\left(\boldsymbol{B}, \boldsymbol{r}_{0}\right), \\
& \boldsymbol{r}_{n} \perp_{\boldsymbol{A}} \boldsymbol{A}^{-1} \boldsymbol{B} \mathcal{K}_{n}\left(\boldsymbol{B}, \boldsymbol{r}_{0}\right), \quad \text { or } \quad \boldsymbol{r}_{n} \perp \boldsymbol{B} \mathcal{K}_{n}\left(\boldsymbol{B}, \boldsymbol{r}_{0}\right) .
\end{aligned}
$$

By comparison with (22) we see that the CGNR method is the minimal residual method with

$$
\boldsymbol{Z}=\boldsymbol{A}, \quad \mathcal{L}_{n}=\boldsymbol{A}^{\top} \mathcal{K}_{n}\left(\boldsymbol{A} \boldsymbol{A}^{\top}, \boldsymbol{r}_{0}\right)=\boldsymbol{A}^{-1} \boldsymbol{B} \mathcal{K}_{n}\left(\boldsymbol{B}, \boldsymbol{r}_{0}\right) .
$$

Note that $\mathcal{L}_{n}$ is of the same form as in (14). CGNR has the following optimality properties:

$$
\begin{aligned}
& \left\|\boldsymbol{b}-\boldsymbol{A} \boldsymbol{x}_{n}\right\|=\min _{\boldsymbol{x} \in \boldsymbol{x}_{0}+\boldsymbol{A}^{\top} \mathcal{K}_{n}\left(\boldsymbol{A} \boldsymbol{A}^{\top}, \boldsymbol{r}_{0}\right)}\|\boldsymbol{b}-\boldsymbol{A} \boldsymbol{x}\|, \\
& \left\|\boldsymbol{x}_{n}-\boldsymbol{A}^{-1} \boldsymbol{b}\right\|_{\boldsymbol{A}^{\top} \boldsymbol{A}}=\min _{\boldsymbol{x} \in \boldsymbol{x}_{0}+\boldsymbol{A}^{\top} \mathcal{K}_{n}\left(\boldsymbol{A} \boldsymbol{A}^{\top}, \boldsymbol{r}_{0}\right)}\left\|\boldsymbol{x}-\boldsymbol{A}^{-1} \boldsymbol{b}\right\|_{\boldsymbol{A}^{\top} \boldsymbol{A}},
\end{aligned}
$$

so it minimizes indeed the 2-norm of the residual over the search space $\boldsymbol{x}_{0}+\mathcal{L}_{n}$.

The second possibility is to apply the CG method to the positive definite system $\boldsymbol{A} \boldsymbol{A}^{\top} \boldsymbol{y}=\boldsymbol{b}$, where $\boldsymbol{x}=\boldsymbol{A}^{\top} \boldsymbol{y}$. This approach leads to the CGNE method of Craig [7] with (33)-(34) as before (and, hence, also (36)-(37)), but with (35) replaced by

$$
\boldsymbol{r}_{n} \perp \mathcal{K}_{n}\left(\boldsymbol{A} \boldsymbol{A}^{\top}, \boldsymbol{r}_{0}\right)=\boldsymbol{A}^{-\top} \boldsymbol{A}^{\top} \mathcal{K}_{n}\left(\boldsymbol{A} \boldsymbol{A}^{\top}, \boldsymbol{r}_{0}\right)
$$

or

$$
\boldsymbol{r}_{n} \perp_{\boldsymbol{A}^{-\top}} \boldsymbol{A}^{\top} \mathcal{K}_{n}\left(\boldsymbol{A} \boldsymbol{A}^{\top}, \boldsymbol{r}_{0}\right)=\boldsymbol{A}^{-1} \boldsymbol{B} \mathcal{K}_{n}\left(\boldsymbol{B}, \boldsymbol{r}_{0}\right)=\mathcal{L}_{n}
$$

Consequently, the CGNE method fits into our framework if

$$
\boldsymbol{Z}=\boldsymbol{A}^{-\top}, \quad \mathcal{L}_{n}=\boldsymbol{A}^{\top} \mathcal{K}_{n}\left(\boldsymbol{A} \boldsymbol{A}^{\top}, \boldsymbol{r}_{0}\right)=\boldsymbol{A}^{-1} \boldsymbol{B} \mathcal{K}_{n}\left(\boldsymbol{B}, \boldsymbol{r}_{0}\right) .
$$

Due to the properties of the CG method applied to a system with symmetric positive definite $\boldsymbol{A A}^{\top}$, the approximate solutions $\boldsymbol{x}_{n}$ of the CGNE method always exist and have the minimization properties

$$
\begin{aligned}
& \left\|\boldsymbol{b}-\boldsymbol{A} \boldsymbol{x}_{n}\right\|_{\left(\boldsymbol{A} \boldsymbol{A}^{\top}\right)^{-1}}=\min _{\boldsymbol{x} \in \boldsymbol{x}_{0}+\boldsymbol{A}^{\top} \mathcal{K}_{n}\left(\boldsymbol{A} \boldsymbol{A}^{\top}, \boldsymbol{r}_{0}\right)}\|\boldsymbol{b}-\boldsymbol{A} \boldsymbol{x}\|_{\left(\boldsymbol{A} \boldsymbol{A}^{\top}\right)^{-1}}, \\
& \left\|\boldsymbol{x}_{n}-\boldsymbol{A}^{-1} \boldsymbol{b}\right\|=\min _{\boldsymbol{x} \in \boldsymbol{x}_{0}+\boldsymbol{A}^{\top} \mathcal{K}_{n}\left(\boldsymbol{A A ^ { \top } , \boldsymbol { r } _ { 0 } )}\right.}\left\|\boldsymbol{x}-\boldsymbol{A}^{-1} \boldsymbol{b}\right\|,
\end{aligned}
$$


so here the 2-norm of the error is minimized over the search space $\boldsymbol{x}_{0}+\mathcal{L}_{n}$.

The concept of error minimization will be examined further in the following section, where we show that the choice $\boldsymbol{Z}=\boldsymbol{A}^{-\top}$ leads to another interesting class of generalized conjugate gradient methods.

\section{Minimal error methods and generalized minimal error methods}

As we have mentioned in the previous section the class of minimal residual methods has the feature of minimizing the 2-norm of the residual $\boldsymbol{r}_{n}$ of the approximate solution over a certain subspace $\mathcal{L}_{n}$. If the matrix $\boldsymbol{A}$ is well-conditioned, the fast convergence of the residuals $\boldsymbol{r}_{n}$ also implies the fast convergence of the the errors $\boldsymbol{e}_{n}$. In many practical applications however, the matrices are ill-conditioned and the link between these two measures for the error is not as tight. It may then be preferable to use methods that minimize directly the 2-norm of the error $\boldsymbol{e}_{n}$ associated with the generated approximate solutions. This approach is, however, not as straightforward as in the previous case. Though the approximate solution $\boldsymbol{x}_{n}$ from (1) satisfying the error minimization condition

$$
\left\|\boldsymbol{x}_{n}-\boldsymbol{A}^{-1} \boldsymbol{b}\right\|=\min _{\boldsymbol{x} \in \boldsymbol{x}_{0}+\mathcal{K}_{n}\left(\boldsymbol{A}, \boldsymbol{r}_{0}\right)}\left\|\boldsymbol{x}-\boldsymbol{A}^{-1} \boldsymbol{b}\right\|
$$

does exist at each iteration step, it cannot be constructed effectively. In fact, when considering error minimizing methods in the general framework (2)-(3), that is,

$$
\left\|\boldsymbol{e}_{n+1}\right\|_{\boldsymbol{Z}}=\min ! \quad \text { subject to } \boldsymbol{x}_{n} \in \boldsymbol{x}_{0}+\mathcal{L}_{n},
$$

it is easily seen by computing the Fréchet-derivative of $\left\|\boldsymbol{e}_{n+1}\right\|_{\boldsymbol{Z}}$ that these methods fit into the framework (2)-(6) of general orthogonal residual methods by choosing $\boldsymbol{Z}=\boldsymbol{A}^{-\top}$; the Galerkin condition (6) becomes

$$
\boldsymbol{r}_{n} \perp \boldsymbol{A}^{-\top} \mathcal{L}_{n} .
$$

If $\mathcal{L}_{n}=\mathcal{K}_{n}\left(\boldsymbol{A}, \boldsymbol{r}_{0}\right)$, (6) takes the two equivalent forms

$$
\boldsymbol{r}_{n} \perp \boldsymbol{A}^{-\top} \mathcal{K}_{n}\left(\boldsymbol{A}, \boldsymbol{r}_{0}\right) \Longleftrightarrow \boldsymbol{e}_{n}=\boldsymbol{x}_{n}-\boldsymbol{A}^{-1} \boldsymbol{b} \perp \mathcal{K}_{n}\left(\boldsymbol{A}, \boldsymbol{r}_{0}\right) .
$$

But these conditions are useless without explicit knowledge of $\boldsymbol{A}^{-1}$ or $\boldsymbol{A}^{-\top}$.

However, error minimization can be attained for different choices of the subspace $\mathcal{L}_{n}$, as we will see below. Before, we point out that Lemma 1 has for $\boldsymbol{Z}=\boldsymbol{A}^{-\top}$ the following corollary.

Corollary 4. For any minimal error method (47) the error vectors $\boldsymbol{e}_{n}$ and residual vectors $\boldsymbol{r}_{n}$ satisfy

$$
\begin{aligned}
& \left\langle\boldsymbol{e}_{n}, \boldsymbol{e}_{n}-\boldsymbol{e}_{0}\right\rangle=0, \\
& \left\|\boldsymbol{e}_{n}\right\|^{2}+\left\|\boldsymbol{e}_{n}-\boldsymbol{e}_{0}\right\|^{2}=\left\|\boldsymbol{e}_{0}\right\|^{2}, \\
& \left\|\boldsymbol{r}_{n}\right\|_{\left(\boldsymbol{A}^{\top} \boldsymbol{A}\right)^{-1}}^{2}+\left\|\boldsymbol{r}_{n}-\boldsymbol{r}_{0}\right\|_{\left(\boldsymbol{A}^{\top} \boldsymbol{A}\right)^{-1}}^{2}=\left\|\boldsymbol{r}_{0}\right\|_{\left(\boldsymbol{A}^{\top} \boldsymbol{A}\right)^{-1}}^{2} .
\end{aligned}
$$

Again, (50) describes a Thales circle, while (51) and (52) are just the corresponding statements of Pythagoras' theorem; see Fig. 2. But here, for minimal error methods, the roles of the residual vectors $\boldsymbol{r}_{n}$ and the error vectors $\boldsymbol{e}_{n}$ are reversed. Now the error $\boldsymbol{e}_{n}$ lies on an $n$-dimensional sphere with the diameter equal to the norm of the initial error $\boldsymbol{e}_{0}$. 

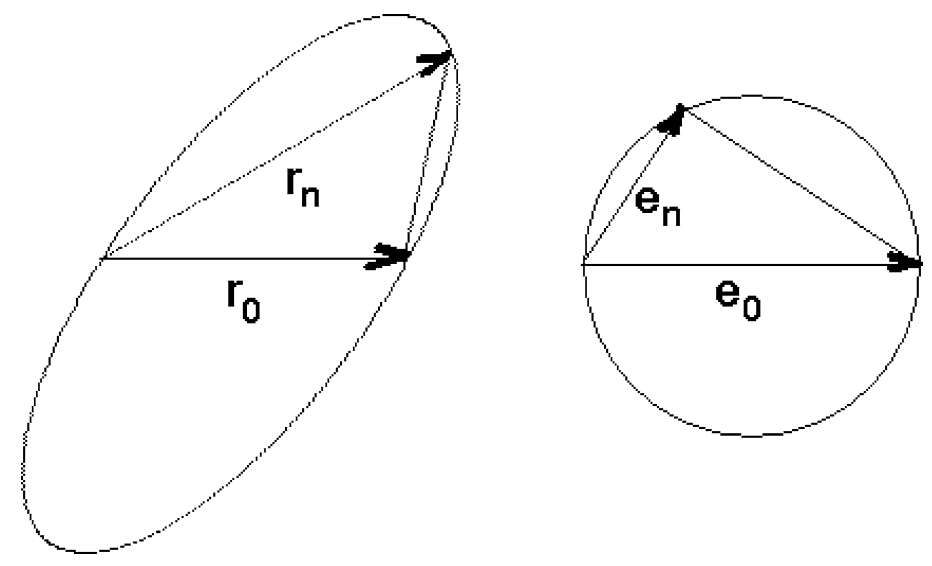

Fig. 2. Geometric properties of minimal error methods.

One of the feasible minimal error methods corresponds to the choice $\mathcal{L}_{n}=\boldsymbol{A}^{\top} \mathcal{K}_{n}\left(\boldsymbol{A}^{\top}, \boldsymbol{r}_{0}\right)$. Then the approximate solutions $\boldsymbol{x}_{n}$ satisfy the conditions

$$
\boldsymbol{x}_{n} \in \boldsymbol{x}_{0}+\boldsymbol{A}^{\top} \mathcal{K}_{n}\left(\boldsymbol{A}^{\top}, \boldsymbol{r}_{0}\right), \quad \boldsymbol{r}_{n} \in \boldsymbol{r}_{0}+\boldsymbol{A} \boldsymbol{A}^{\top} \mathcal{K}_{n}\left(\boldsymbol{A}^{\top}, \boldsymbol{r}_{0}\right), \quad \boldsymbol{r}_{n} \perp \mathcal{K}_{n}\left(\boldsymbol{A}^{\top}, \boldsymbol{r}_{0}\right) .
$$

These approximations always exist and are uniquely determined. It can be verified easily that the conditions (53) are equivalent to the error minimization properties

$$
\begin{aligned}
& \left\|\boldsymbol{b}-\boldsymbol{A} \boldsymbol{x}_{n}\right\|_{\left(\boldsymbol{A}^{\top} \boldsymbol{A}\right)^{-1}}=\min _{\boldsymbol{x} \in \boldsymbol{x}_{0}+\boldsymbol{A}^{\top} \mathcal{K}_{n}\left(\boldsymbol{A}^{\top}, \boldsymbol{r}_{0}\right)}\|\boldsymbol{b}-\boldsymbol{A} \boldsymbol{x}\|_{\left(\boldsymbol{A}^{\top} \boldsymbol{A}\right)^{-1},} \\
& \left\|\boldsymbol{x}_{n}-\boldsymbol{A}^{-1} \boldsymbol{b}\right\|=\min _{\boldsymbol{x} \in \boldsymbol{x}_{0}+\boldsymbol{A}^{\top} \mathcal{K}_{n}\left(\boldsymbol{A}^{\top}, \boldsymbol{r}_{0}\right)}\left\|\boldsymbol{x}-\boldsymbol{A}^{-1} \boldsymbol{b}\right\| .
\end{aligned}
$$

Consequently, the error norms decrease monotonously and, therefore, converge. However, this does not mean that they need to converge to zero, and, there is no guarantee that the approximations $\boldsymbol{x}_{n}$ converge to the solution $\boldsymbol{A}^{-1} \boldsymbol{b}$. In fact, when the Krylov space generated by $\boldsymbol{A}^{\top}$ is exhausted, that is, when $\mathcal{K}_{m+1}\left(\boldsymbol{A}^{\top}, \boldsymbol{r}_{0}\right)=\mathcal{K}_{m}\left(\boldsymbol{A}^{\top}, \boldsymbol{r}_{0}\right)$, we have now in contrast to (8)

$$
\mathcal{K}_{m}\left(\boldsymbol{A}^{\top}, \boldsymbol{r}_{0}\right) \perp \boldsymbol{r}_{m} \in \boldsymbol{r}_{0}+\boldsymbol{A} \mathcal{K}_{m}\left(\boldsymbol{A}^{\top}, \boldsymbol{r}_{0}\right) \not \subset \mathcal{K}_{m+1}\left(\boldsymbol{A}^{\top}, \boldsymbol{r}_{0}\right)=\mathcal{K}_{m}\left(\boldsymbol{A}^{\top}, \boldsymbol{r}_{0}\right),
$$

and this does not imply that $\boldsymbol{r}_{m}=\boldsymbol{o}$. So, in general, GMERR does not converge to the exact solution, but it ultimately stagnates with some nonzero residual; see also [10].

For symmetric matrices $\boldsymbol{A}$ we do not have this problem. For such systems the minimal error method defined by (53) is frequently used. It is known under several names and comes in various mathematically equivalent schemes, which may substantially differ in their algorithmic formulation and their numerical behavior. The first minimal error method for symmetric systems was the orthogonal direction (OD) method due to Fridman [16]. Another, breakdown-free formulation of this method was introduced by Fletcher [13], and its stable form, the STOD method, was proposed by Stoer [32]. Another numerically stable algorithm for symmetric indefinite systems, SYMMLQ, was introduced by Paige and Saunders [25]. It makes use of the symmetric Lanczos process for computing an orthonormal basis of $\mathcal{K}_{n}\left(\boldsymbol{A}, \boldsymbol{r}_{0}\right)$. These symmetric error minimizing algorithms were carefully reviewed by Freund [14], who 
also presented yet another stable implementation of the symmetric indefinite minimal error method called ME.

Error minimization for general nonsymmetric systems was proposed by Weiss in [39]; see also [41]. He introduced the generalized minimal error method (GMERR) and showed that in some applications restarted GMERR was very efficient. The numerical properties of the original implementation of GMERR were discussed in [27], where also more stable implementations and variants were proposed. Ehrig and Deuflhard [10] derived another implementation of the GMERR method and discussed the limitations for its use in the general nonsymmetric case.

\section{The connection between orthogonal residual and minimal residual methods}

In this section we denote the iterates and the residuals of an orthogonal residual method by $\boldsymbol{x}_{n}$ and $\boldsymbol{r}_{n}$, and those of the corresponding minimum residual method (using the same search space and the same formal inner product matrix $\boldsymbol{Z}$ ) by $\widetilde{\boldsymbol{x}}_{n}$ and $\widetilde{\boldsymbol{r}}_{n}$. Our aim is to discuss the connections between pairs of such methods. These connections were revealed by Weiss in his thesis [37].

According to (30) and (26) the orthogonal residual $\boldsymbol{r}_{n}$ (if it exists) and the minimizing residuals $\widetilde{\boldsymbol{r}}_{n}$ and $\widetilde{\boldsymbol{r}}_{n-1}$ satisfy the conditions

$$
\boldsymbol{r}_{n}, \widetilde{\boldsymbol{r}}_{n} \in \boldsymbol{r}_{0}+\boldsymbol{A} \mathcal{L}_{n} \subseteq \mathcal{L}_{n+1}, \quad \widetilde{\boldsymbol{r}}_{n-1} \in \boldsymbol{r}_{0}+\boldsymbol{A} \mathcal{L}_{n-1} \subseteq \mathcal{L}_{n} \subseteq \mathcal{L}_{n+1}
$$

and

$$
\boldsymbol{r}_{n} \perp \mathcal{L}_{n}, \quad \widetilde{\boldsymbol{r}}_{n} \perp \boldsymbol{A} \mathcal{L}_{n}, \quad \widetilde{\boldsymbol{r}}_{n-1} \perp \boldsymbol{A} \mathcal{L}_{n-1} .
$$

Consequently, the differences

$$
\boldsymbol{r}_{n}-\widetilde{\boldsymbol{r}}_{n-1}, \widetilde{\boldsymbol{r}}_{n}-\widetilde{\boldsymbol{r}}_{n-1} \in \boldsymbol{A} \mathcal{L}_{n} \ominus \boldsymbol{A} \mathcal{L}_{n-1}
$$

lie in a one-dimensional subspace, so normally each one will be a scalar multiple of the other. Indeed, it can be shown that whenever $\boldsymbol{r}_{n}$ exists, there are recursions of the form

$$
\tilde{\boldsymbol{x}}_{n}:=\widetilde{\boldsymbol{x}}_{n-1}\left(1-\theta_{n}\right)+\boldsymbol{x}_{n} \theta_{n}, \quad \widetilde{\boldsymbol{r}}_{n}:=\widetilde{\boldsymbol{r}}_{n-1}\left(1-\theta_{n}\right)+\boldsymbol{r}_{n} \theta_{n} .
$$

The coefficients $\theta_{n}$ can be found by making $\left\|\widetilde{\boldsymbol{r}}_{n}\right\|$ minimal in (60), that is, by imposing

$$
\widetilde{\boldsymbol{r}}_{n} \perp \widetilde{\boldsymbol{r}}_{n-1}-\boldsymbol{r}_{n} \in \boldsymbol{A} \mathcal{L}_{n}
$$

Therefore, if we let

$$
\theta_{n}:=\frac{\left\langle\widetilde{\boldsymbol{r}}_{n-1}-\boldsymbol{r}_{n}, \widetilde{\boldsymbol{r}}_{n-1}\right\rangle}{\left\|\widetilde{\boldsymbol{r}}_{n-1}-\boldsymbol{r}_{n}\right\|^{2}},
$$

then the recursions (60) allow us to construct the sequences $\left\{\widetilde{\boldsymbol{x}}_{n}\right\}$ and $\left\{\widetilde{\boldsymbol{r}}_{n}\right\}$ from the sequences $\left\{\boldsymbol{x}_{n}\right\}$ and $\left\{\boldsymbol{r}_{n}\right\}$. For a geometric interpretation see Fig. 3, which is taken from [19]. In other words, if we compute the orthogonal residual approximations and residuals, then we can obtain from them the minimal residual approximations and residuals nearly for free.

In retrospect this is a simple, but nevertheless quite surprising result obtained by Weiss in his thesis [37,38] where he analyzed Schönauer's smoothing method [30], which consists of applying the recursions (60) with $\theta_{n}$ given by (62) to any sequences $\left\{\boldsymbol{x}_{n}\right\}$ and $\left\{\boldsymbol{r}_{n}\right\}$ constructed by an iterative method. 


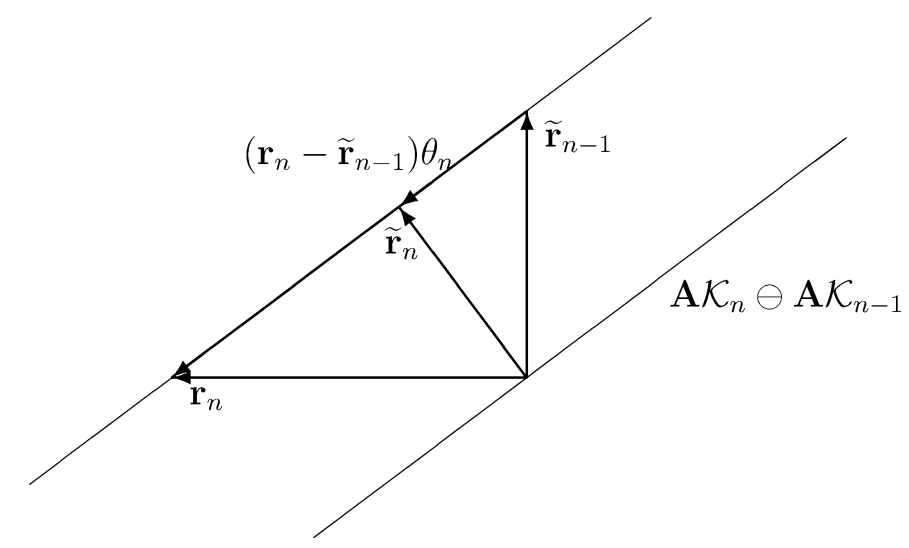

Fig. 3. Relationship between minimal residual methods and orthogonal residual methods: the 2-dimensional space $\mathcal{K}_{n+1} \ominus \boldsymbol{A} \mathcal{K}_{n-1}$ containing $\boldsymbol{r}_{n}, \widetilde{\boldsymbol{r}}_{n}, \widetilde{\boldsymbol{r}}_{n-1}$.

Weiss derived for the above situation of an orthogonal set $\left\{\boldsymbol{r}_{n}\right\}$ also a number of relationships between this set and $\left\{\widetilde{\boldsymbol{r}}_{n}\right\}$. For example,

$$
\frac{1}{\left\|\widetilde{\boldsymbol{r}}_{n}\right\|^{2}}=\frac{1}{\left\|\widetilde{\boldsymbol{r}}_{n-1}\right\|^{2}}+\frac{1}{\left\|\boldsymbol{r}_{n}\right\|^{2}}=\sum_{k=0}^{n} \frac{1}{\left\|\boldsymbol{r}_{k}\right\|^{2}}
$$

holds, a relation that was instrumental for understanding the so-called peak-plateau connection $[8,9,35]$, that is, the fact that a peak in the residual norm history of an orthogonal residual method is accompanied by a plateau (where the norm stagnates) in the residual norm history of the corresponding minimal residual method.

\section{Theory of minimal residual smoothing}

The main results on residual smoothing can be developed in another way that can be viewed as a generalization of the approach discussed in the previous section. In this section we review the results on (minimal) residual smoothing obtained originally by Weiss in [37]; see also [40] and [41]. Weiss did not only consider the standard minimal residual and orthogonal residual methods (57) and (58), but made his investigations in the general framework of smoothing processes.

Throughout the section we use in accordance with the previous section the following notation: the iterates, residuals, and error vectors of the primary (unsmoothed) method are denoted $\boldsymbol{x}_{n}, \boldsymbol{r}_{n}$, and $\boldsymbol{e}_{n}$, respectively, while the corresponding smoothed quantities are referred to as $\widetilde{\boldsymbol{x}}_{n}, \widetilde{\boldsymbol{r}}_{n}$, and $\widetilde{\boldsymbol{e}}_{n}$. By definition of the minimal residual (MR) smoothing process these iterates and residuals are related by

$$
\begin{array}{ll}
\widetilde{\boldsymbol{r}}_{0}=\boldsymbol{r}_{0}, & \widetilde{\boldsymbol{r}}_{n}=\widetilde{\boldsymbol{r}}_{n-1}\left(1-\theta_{n}\right)+\boldsymbol{r}_{n} \theta_{n}, \\
\widetilde{\boldsymbol{x}}_{0}=\boldsymbol{x}_{0}, & \widetilde{\boldsymbol{x}}_{n}=\widetilde{\boldsymbol{x}}_{n-1}\left(1-\theta_{n}\right)+\boldsymbol{x}_{n} \theta_{n},
\end{array}
$$

where the smoothing parameter $\theta_{n}$ is defined as the solution of the one-dimensional minimization problem

$$
\left\|\widetilde{\boldsymbol{r}}_{n}\left(\theta_{n}\right)\right\|=\min _{\theta}\left\|\widetilde{\boldsymbol{r}}_{n-1}+\theta\left(\boldsymbol{r}_{n}-\widetilde{\boldsymbol{r}}_{n-1}\right)\right\| .
$$


This approach can be applied to any iterative method, not only to orthogonal residual methods. The minimum is attained when $\widetilde{\boldsymbol{r}}_{n} \perp \boldsymbol{r}_{n}-\widetilde{\boldsymbol{r}}_{n-1}$ as in (61), so $\theta_{n}$ is again given by (62). Weiss showed that this smoothing process transforms any orthogonal residual methods (defined with respect to an spd inner product matrix $\boldsymbol{Z}$ ) into the corresponding minimal residual method. This is in accordance with our results in the previous section, obtained from a different point of view. Geometric properties of the smoothed residual vectors $\widetilde{\boldsymbol{r}}_{n}$ resulting from this process when $\boldsymbol{Z}=\boldsymbol{I}$ are given in the following theorem from [37, Theorem 4.4, pp. 80-81].

Theorem 5. Given the iterates and residuals from any orthogonal residual method satisfying

$$
\boldsymbol{r}_{n} \perp \mathcal{L}_{n} \quad \text { subject to } \boldsymbol{x}_{n} \in \boldsymbol{x}_{0}+\mathcal{L}_{n},
$$

the smoothed residuals $\widetilde{\boldsymbol{r}}_{n}$ obtained from (64) satisfy

$$
\left\langle\widetilde{\boldsymbol{r}}_{n}, \widetilde{\boldsymbol{r}}_{n}-\widetilde{\boldsymbol{r}}_{0}\right\rangle=0
$$

Indeed, when applying the minimal residual smoothing as a postprocessing of the orthogonal residual method, the smoothed residual lies on the $n$-dimensional sphere with the radius equal to $\left\|\boldsymbol{r}_{0}\right\| / 2$. In other words, Fig. 1 holds also for $\widetilde{\boldsymbol{r}}_{0}, \widetilde{\boldsymbol{r}}_{n}, \widetilde{\boldsymbol{e}}_{0}$, and $\widetilde{\boldsymbol{e}}_{n}$. This result is natural from our previous point of view, since the smoothed quantities are identical to those of the minimal residual method. Thus the statements of Corollary 3 hold and take now the form

$$
\begin{aligned}
& \left\langle\widetilde{\boldsymbol{r}}_{n}, \widetilde{\boldsymbol{r}}_{n}-\widetilde{\boldsymbol{r}}_{0}\right\rangle=0, \\
& \left\|\widetilde{\boldsymbol{r}}_{n}\right\|^{2}+\left\|\widetilde{\boldsymbol{r}}_{n}-\widetilde{\boldsymbol{r}}_{0}\right\|^{2}=\left\|\widetilde{\boldsymbol{r}}_{0}\right\|^{2}, \\
& \left\|\widetilde{\boldsymbol{e}}_{n}\right\|_{\boldsymbol{A}^{\top} \boldsymbol{A}}^{2}+\left\|\widetilde{\boldsymbol{e}}_{n}-\widetilde{\boldsymbol{e}}_{0}\right\|_{\boldsymbol{A}^{\top} \boldsymbol{A}}^{2}=\left\|\widetilde{\boldsymbol{e}}_{0}\right\|_{\boldsymbol{A}^{\top} \boldsymbol{A}}^{2} .
\end{aligned}
$$

The situation is much less understood when the minimal residual smoothing process is applied to primary methods that do not have orthogonal residuals. An example of such a method is the popular and very effective biconjugate gradient (BICG) method of Lanczos [23] and Fletcher [13]. Its iterates $\boldsymbol{x}_{n}$ satisfy

$$
\boldsymbol{x}_{n} \in \boldsymbol{x}_{0}+\mathcal{K}_{n}\left(\boldsymbol{A}, \boldsymbol{r}_{0}\right), \quad \boldsymbol{r}_{n} \perp \mathcal{K}_{n}\left(\boldsymbol{A}^{\top}, \boldsymbol{r}_{0}\right) .
$$

As pointed out repeatedly by Weiss and others this method fits formally into the general framework when we replace $\boldsymbol{A}$ and $\boldsymbol{Z}$ by

$$
\mathcal{A}:=\left[\begin{array}{cc}
\boldsymbol{O} & \boldsymbol{A} \\
\boldsymbol{A}^{\top} & \boldsymbol{O}
\end{array}\right], \quad \mathcal{Z}:=\left[\begin{array}{cc}
\boldsymbol{O} & \boldsymbol{I} \\
\boldsymbol{I} & \boldsymbol{O}
\end{array}\right] .
$$

However, this $\mathcal{Z}$ is not spd, and therefore most of the results derived do not hold here. In fact, theoretically this case is very different from the one treated in this paper.

When we apply the minimal residual smoothing (64)-(66) to the iterates and residuals of the BICG method characterized by (67), we still obtain smoothed quantities. This combination is called the BICO method by Schönauer and Weiss; see [41]. It is not equivalent to the quasi-minimal residual (QMR) method of Freund and Nachtigal [15], but a different choice of parameter in the smoothing process leads to an analogue smoothing process called quasi-minimal residual (QMR) smoothing [43], which, when applied to BICG, yields theoretically the QMR iterates and residuals. This connection can be used to understand why the following result, which is very similar to one for the QMR method [15], holds for 
the BICO method. It follows, e.g., from Theorem 5.8 in Weiss' book [41], that the norm of the BICO residual is bounded by

$$
\left\|\boldsymbol{b}-\boldsymbol{A} \widetilde{\boldsymbol{x}}_{n}\right\| \leqslant \sqrt{n+1} \frac{1}{\sqrt{\sum_{k=0}^{n} \frac{1}{\left\|\boldsymbol{r}_{k}\right\|^{2}}}},
$$

where the quantity $\sqrt{\sum_{k=0}^{n} \frac{1}{\left\|\boldsymbol{r}_{k}\right\|^{2}}}$ is the norm of the so-called quasi-residual, which plays also an important role in the QMR method [35]. For a thorough analysis of residual smoothing techniques we refer to Chapter 5 of [41].

\section{References}

[1] S.F. Ashby, M.H. Gutknecht, A matrix analysis of conjugate gradient algorithms, in: M. Natori, T. Nodera (Eds.), Advances in Numerical Methods for Large Sparse Sets of Linear Systems, Parallel Processing for Scientific Computing, Vol. 9, Keio University, Yokahama, Japan, 1993, pp. 32-47.

[2] S.F. Ashby, T.A. Manteuffel, P.E. Saylor, A taxonomy for conjugate gradient methods, SIAM J. Numer. Anal. 27 (1990) 1542-1568.

[3] O. Axelsson, Conjugate gradient type methods for unsymmetric and inconsistent systems of linear equations, Linear Algebra Appl. 29 (1980) 1-16.

[4] P.N. Brown, A theoretical comparison of the Arnoldi and GMRES algorithms, SIAM J. Sci. Statist. Comput. 12 (1991) 58-78.

[5] R. Chandra, Conjugate gradient methods for partial differential equations, Ph.D. Thesis, Yale University, New Haven, CT, Technical Report 129, 1978.

[6] P. Concus, G.H. Golub, A generalized conjugate gradient method for nonsymmetric systems of linear equations, in: R. Glowinski, J. Lions (Eds.), Computing Methods in Applied Sciences and Engineering, Lecture Notes in Economics and Math. Syst., Vol. 134, Springer, Berlin, 1976, pp. 56-65.

[7] E.J. Craig, The $n$-step iteration procedure, J. Math. Phys. 34 (1955) 64-73.

[8] J. Cullum, Peaks, plateaus, numerical instabilities in a Galerkin/minimal residual pair of methods for solving $A x=b$, Appl. Numer. Math. 19 (3) (1995) 255-278.

[9] J. Cullum, A. Greenbaum, Relations between Galerkin and norm-minimizing iterative methods for solving linear systems, SIAM J. Matrix Anal. Appl. 17 (2) (1996) 223-247.

[10] R. Ehrig, P. Deuflhard, GMERR — an error minimizing variant of GMRES, Preprint SC 97-63, Konrad-ZuseZentrum für Informationstechnik, Berlin, 1997.

[11] S.C. Eisenstat, H.C. Elman, M.H. Schultz, Variational iterative methods for nonsymmetric systems of linear equations, SIAM J. Numer. Anal. 20 (2) (1983) 345-357.

[12] H.C. Elman, Iterative methods for large, sparse, nonsymmetric systems of linear equations, Ph.D. Thesis, Computer Science Department, Yale University, New Haven, CT, 1982.

[13] R. Fletcher, Conjugate gradient methods for indefinite systems, in: G.A. Watson (Ed.), Numerical Analysis, Dundee, 1975, Lecture Notes in Math., Vol. 506, Springer, Berlin, 1976, pp. 73-89.

[14] R.W. Freund, On conjugate gradient type methods and polynomial preconditioners for a class of complex non-Hermitian matrices, Numer. Math. 57 (1990) 285-312.

[15] R.W. Freund, N.M. Nachtigal, QMR: A quasi-minimal residual method for non-Hermitian linear systems, Numer. Math. 60 (1991) 315-339.

[16] V.M. Fridman, The method of minimum iterations with minimum errors for a system of linear algebraic equations with a symmetrical matrix, USSR Comput. Math. Math. Phys. 2 (1963) 362-363. 
[17] M.H. Gutknecht, Changing the norm in conjugate gradient type algorithms, SIAM J. Numer. Anal. 30 (1993) $40-56$.

[18] M.H. Gutknecht, Lanczos-type solvers for nonsymmetric linear systems of equations, Acta Numer. 6 (1997) 271-397.

[19] M.H. Gutknecht, M. Rozložník, By how much can residual minimization accelerate the convergence of orthogonal residual methods?, Numer. Algor. 27 (2001) 189-213.

[20] L.A. Hageman, D.M. Young, Applied Iterative Methods, Academic Press, Orlando, FL, 1981.

[21] M.R. Hestenes, E. Stiefel, Methods of conjugate gradients for solving linear systems, J. Res. Nat. Bureau Standards 49 (1952) 409-435.

[22] I.M. Khabaza, An iterative least-square method suitable for solving large sparse matrices, Comput. J. 6 (1963/1964) 202-206.

[23] C. Lanczos, Solution of systems of linear equations by minimized iterations, J. Res. Nat. Bureau Standards 49 (1952) 33-53.

[24] D.G. Luenberger, Hyperbolic pairs in the method of conjugate gradients, SIAM J. Appl. Math. 17 (1979) $1263-1267$.

[25] C.C. Paige, M.A. Saunders, Solution of sparse indefinite systems of linear equations, SIAM J. Numer. Anal. 12 (1975) 617-629.

[26] M. Rozložník, Z. Strakoš, Variants of the residual minimizing Krylov space methods, in: I. Marek (Ed.), Proceedings of the XI Summer School "Software and Algorithms in Numerical Mathematics", University of West Bohemia, 1995, pp. 208-225.

[27] M. Rozložník, R. Weiss, On the stable implementation of the generalized minimal error method, J. Comput. Appl. Math. 98 (1998) 49-62.

[28] Y. Saad, Practical use of some Krylov subspace methods for solving indefinite and nonsymmetric linear systems, SIAM J. Sci. Statist. Comput. 5 (1984) 203-228.

[29] Y. Saad, M.H. Schultz, GMRES: A generalized minimal residual algorithm for solving nonsymmetric linear systems, SIAM J. Sci. Statist. Comput. 7 (1986) 856-869.

[30] W. Schönauer, Scientific Computing on Vector Computers, Elsevier, Amsterdam, 1987.

[31] E. Stiefel, Relaxationsmethoden bester Strategie zur Lösung linearer Gleichungssysteme, Comment Math. Helv. 29 (1955) 157-179.

[32] J. Stoer, Solution of large linear systems of equations by conjugate gradient methods, in: A. Bachen, M. Grötschel, B. Korte (Eds.), Mathematical Programming-The State of the Art-Bonn 1982, Springer, Berlin, 1983, pp. 540-565.

[33] P.K.W. Vinsome, ORTHOMIN — an iterative method for solving sparse sets of simultaneous linear equations, in: Proc. Fourth SPE Symposium on Reservoir Simulation, Los Angeles, CA, 1976, pp. 149-160.

[34] H.F. Walker, Implementations of the GMRES method, Comput. Phys. Comm. 53 (1989) 311-320.

[35] H.F. Walker, Residual smoothing and peak/plateau behavior in Krylov subspace methods, Appl. Numer. Math. 19 (1995) 279-286.

[36] H.F. Walker, L. Zhou, A simpler GMRES, Research Report 10/92/54, Department of Mathematics and Statistics, Utah State University, Logan, UT, 1992.

[37] R. Weiss, Convergence behavior of generalized conjugate gradient methods, Ph.D. Thesis, University of Karlsruhe, 1990.

[38] R. Weiss, Properties of generalized conjugate gradient methods, J. Numer. Linear Algebra Appl. 1 (1) (1994) 45-63.

[39] R. Weiss, Error-minimizing Krylov subspace methods, SIAM J. Sci. Comput. 15 (1994) 511-527.

[40] R. Weiss, Minimization properties and short recurrences for Krylov subspace methods, Electronic Trans. Numer. Anal. 2 (1994) 57-75.

[41] R. Weiss, Parameter-Free Iterative Linear Solvers, Mathematical Research, Vol. 97, Akademie Verlag, Berlin, 1996. 
[42] D.M. Young, K.C. Jea, Generalized conjugate-gradient acceleration of nonsymmetrizable iterative methods, Linear Algebra Appl. 34 (1980) 159-194.

[43] L. Zhou, H.F. Walker, Residual smoothing techniques for iterative methods, SIAM J. Sci. Comput. 15 (1994) 297-312. 